\title{
Integrating PARP inhibitors into the management of breast cancer: where are we?
}

\author{
Hamdy A. Azim', Loay Kassem ${ }^{1}$, Hatem Azim Jr ${ }^{2}$ \\ ${ }^{1}$ Clinical Oncology Department, KasrAlainy School of Medicine, Cairo University, Cairo, Egypt; ${ }^{2}$ Department of Medicine, Division of Hematology- \\ Oncology, American University of Beirut, Beirut, Lebanon \\ Contributions: (I) Conception and design: HA Azim, H Azim Jr; (II) Administrative support: L Kassem; (III) Provision of study materials or patients: \\ HA Azim; (IV) Collection and assembly of data: All authors; (V) Data analysis and interpretation: HA Azim, L Kassem; (VI) Manuscript writing: All \\ authors; (VII) Final approval of manuscript: All authors. \\ Correspondence to: Loay Kassem. Cairo University Hospitals, Alsaray St., Elmanial, Cairo 11451, Egypt. Email: loay.kassem@cairocure.com.
}

\begin{abstract}
During the last 2 decades, extensive research has focused on the molecular functions of BRCA1 and BRCA2 genes. This has led to the development of Poly(ADP-ribose) polymerase inhibitors (PARPi), as effective target therapies, based on their preferential cytotoxicity in tumor cells harboring germline BRCA1 and BRCA2 mutations. At the present time, 2 PARPi (Olaparib and Talazoparib) are approved as single agent for the treatment of patients with metastatic HER2-ve breast cancer, who have BRCA germline mutations. The clinical benefit of these agents might be also anticipated in patients harboring germline mutations in some additional genes involved in the process of homologous recombination repair (HRR) other than BRCA1/BRCA2. In this review, we summarize the molecular rational for the therapeutic development of PARPi and the clinical evidence supporting their use as anticancer drugs in breast cancer patients with BRCA1/BRCA2 germline mutations. We also discuss the role of platinum-based chemotherapy and how it compares with PARPi in the management of these patients. We will go through some relevant clinical trials of various combinations of PARPi with cytotoxic or immunotherapeutic agents, which may potentially provide better treatment results, compared to what is already achieved with their use as monotherapy.
\end{abstract}

Keywords: Breast cancer; PARP inhibitors; BRCA mutation; DNA repair

Submitted Nov 11, 2019. Accepted for publication Oct 19, 2020.

doi: $10.21037 / \mathrm{cco}-19-230$

View this article at: http://dx.doi.org/10.21037/cco-19-230

\section{Introduction}

Approximately $5-10 \%$ of breast cancers are secondary to inherited mutations (1). Within this group, the vast majority is caused by mutations in the tumour suppressor genes BRCA1 and BRCA2. BRCA1-mutation carriers have a lifetime risk of up to $85 \%$ to develop breast cancer and about $45 \%$ for ovarian cancer, while those harboring BRCA2 germline mutations have a lifetime risk of around $66 \%$ and $12 \%$ to develop breast cancer and ovarian cancer, respectively $(2,3)$. BRCA2 mutations also predispose to cancers of the male breast, pancreas, prostate and other organs.

BRCA1-mutated breast cancers are frequently triple negative (only $~ 10 \%$ are HER2-positive) and show a higher mitotic index and increased lymphocytic infiltration compared to sporadic cancers. Moreover, they generally exhibit basal-like characteristics, defined by the expression of genes specific to the basal mammary myoepithelial cells (4-6). Conversely, $77 \%$ of breast tumors arising in BRCA2 mutation carriers are ER-positive and only $15 \%$ are triple negative, largely mimicking the general population in terms of breast cancer phenotypes $(4,6,7)$.

In spite of these epidemiologic and phenotypic dissimilarities related to tumors associated with BRCA1 and BRCA2 mutations, still the loss of function of any of these 2 genes can lead to one common genetic outcome, known as defective DNA homologous recombination repair (HRR). 
This in turn results in frequent accumulation of genetic mutations, which would ultimately increase susceptibly to develop breast cancer and ovarian cancer and hence the term hereditary breast and ovarian cancer (HBOC) syndrome $(2,8,9)$.

\section{BRCA proteins and DNA damage response (DDR)}

The human cells are spontaneously exposed to thousands of endogenous and exogenous DNA damaging events on daily basis. These episodes result in various DNA aberrations that are normally repaired by multiple repair pathways, collectively known as DDR (10). In general, the double strand breaks (DSBs) are the most hazardous events and they are repaired by either non-homologous end joining (NHEJ) process, or HRR (11). Although the NHEJ provides a rapid and simple pathway to repair DSBs, still it is a low fidelity repair system, which is associated with genomic instability, that may ultimately lead to either cell death, or cell survival with accumulation of genetic mutations and high susceptibility for cancer development $(10,11)$.

On the other hand, the HRR is an error-free pathway, that can perfectly restore the genomic sequence of the broken DNA ends by using the sister chromatid as template for DNA repair (11). However, the HRR is an extremely complex pathway, that needs the interplay of intact BRCA1 and BRCA2 proteins, in addition to several other proteins, (e.g., RAD51C, RAD51D, ATM, BARD1, CHEK2, PALB2, and BRIP1) in order to be concluded (12-14) (Figure 1). Consequences HRR deficiency (HRD) were first described in BRCA1/2 mutant cells, where the error-prone NHEJ pathway takes the lead in repairing DSBs, and hence the high incidence of cancer in patients harboring germline mutations in these 2 genes $(7,11,15)$.

Conversely, DNA single-strand breaks (SSBs) are repaired by base excision repair (BER), primarily conducted by the PARP1 and PARP2 enzymes [poly(adenosine diphosphate ribose) polymerase]. PARP-1 is the main enzyme responsible for BER, while PARP2 is less abundant being responsible for only $5 \%$ to $10 \%$ of the total PARP activity. After binding to a site of SSB, PARP uses NAD+ (nicotinamide-adenine-dinucleotide) to generate PAR [poly adenosine diphosphate ribose] polymers on its self (auto-PARylation) $(16,17)$. The formation of PAR chains also appears to play a role in multiple other cellular tasks, including contribution to DSB repair mediated via the NHEJ process. Because of the high negative charge of PAR polymers, extensive auto-PARylation, leads to dissociation of the PARP enzyme from DNA. This last step enables other repair proteins to localize to the DNA lesion and form the BER complex, which ultimately repairs SSB. Notably, when PARP enzyme does nor dissociate from the DNA, the whole process of BER is severely impaired $(18,19)$.

Despite its important role in the cellular response to genotoxic stress, PARP is not required for cell survival. In preclinical models, PARP deficient mice were shown to be viable and significantly protected from development of early onset cancers. Nevertheless, PARP1-deficient mice do certainly exhibit defective DNA SSB repair, which when encountered by DNA replication forks, will be transformed into DSBs, that need to be only repaired by HRR (rather than NHEJ) (20-22).

\section{PARP inhibition and the concept of synthetic lethality}

Two seminal preclinical studies could unequivocally show that BRCA1/2 dysfunction profoundly sensitizes cells to the inhibition of PARP enzymatic activity, resulting in cell cycle arrest and subsequent apoptosis, while it had practically no therapeutic effect in BRCA normal cells. This would indicate a synthetic lethal interaction between PARP and BRCA1/BRCA2 proteins, where PARP inhibition is exclusively cytotoxic in cells harboring BRCA mutations (Figure 1). According to the concept of synthetic lethality, cell death would be efficiently induced by simultaneous loss of function of the 2 key pathways required for cell survival, i.e., pharmacological inhibition of PARP pathway in tumor cells coupled with impairment of the HRR pathway due to BRCA mutations in these cells $(15,23)$. In an analogous context, platinum agents are known to cause DNA crossbreaks that inhibits cellular DNA repair. Like the situation with PARP inhibitors, HRR is also needed to repair DNA lesions caused by platinums. Furthermore, synthetic lethality has been demonstrated in vitro, where BRCA deficient BC cells, have been shown to respond dramatically to platinums, while BRCA intact cells were much less sensitive $(24,25)$.

\section{PARP inhibitors}

The unique vulnerability of BRCA-mutant tumors to the cytotoxic effects of PARP enzymatic blockade has prompted the clinical development of PARP inhibitors as a genotypespecific treatment for a variety of cancers which harbor 


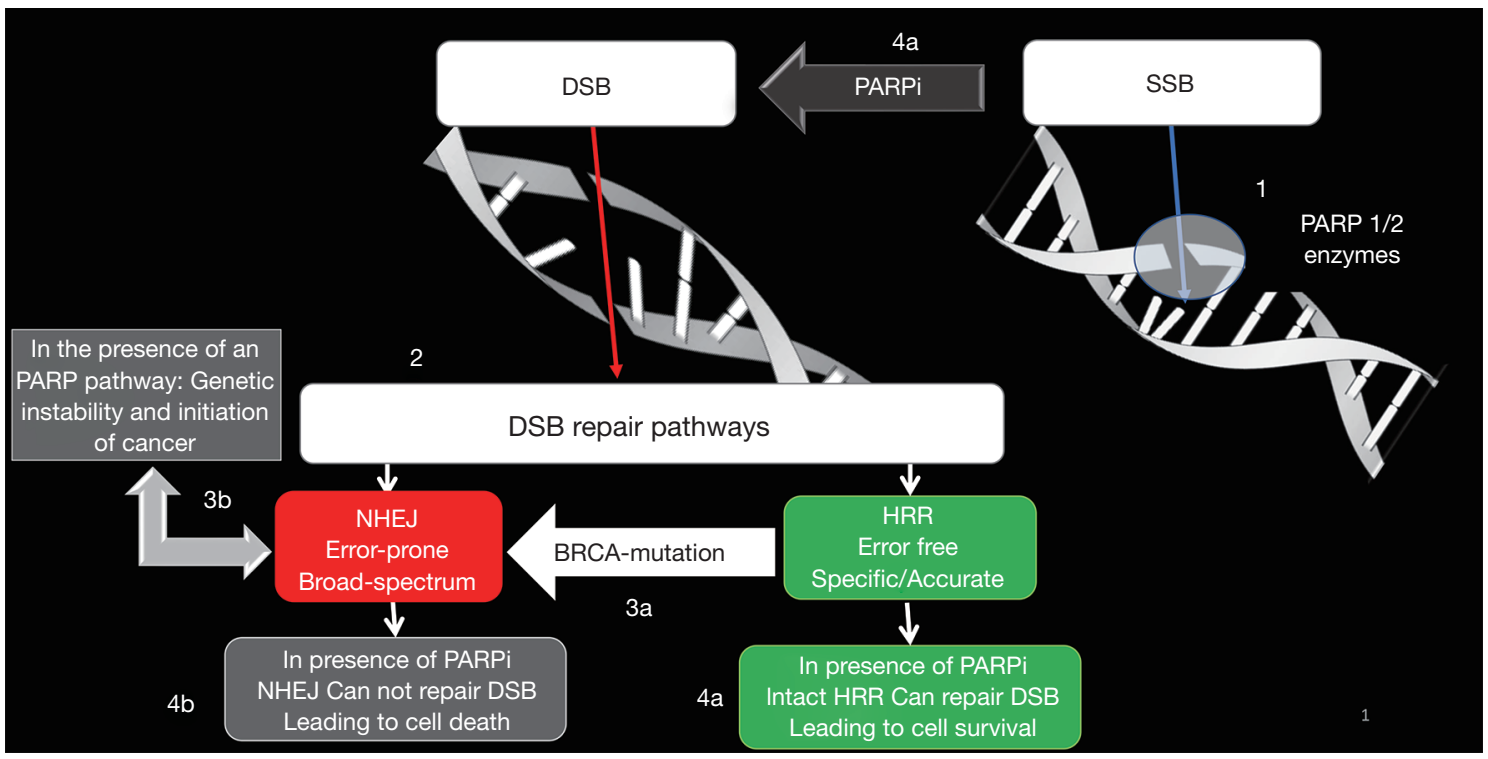

Figure 1 What happens in tumor cells with germline BRCA mutation when treated by a PARPi? 1 and 2: normal cells repair SSBs by PARP enzymatic activity and repair DSB by two mechanisms: HRR and NHEJ. HRR pathway does not depend on PARP enzymatic activity, while NHEJ repair pathway requires an intact PARP pathway; 3a: in the presence of germline BRCA mutation: DSBs are repaired primarily via NHEJ which is highly error prone. $3 \mathrm{~b}$ : this in turn can lead to genetic instability and initiation of BC \& OC. 4a: in BRCA wild cells, treatment with PARPi blocks repair of SSBs, which when encountered by DNA replication forks, will be transformed into DSBs, that are exclusively repaired by HRR (rather than NHEJ), and hence the cells will survive; 4b: in BRCA mutant tumor cells, treatment with PARPi also blocks repair of SSBs, however the 2 pathways responsible for repairing DSBs are out of function. This will end up by accumulation of DSBs, irreversible DNA damage and selective death of BRCA mutant tumor (synthetic lethality).

HRD especially in patients with HBOC syndrome. It should be emphasized that tumor cells arising in carriers of BRCA mutations lack a wild-type copy of BRCA1 or BRCA2, while a single wild-type copy of the relevant gene is still retained by the normal tissues of these patients (26). Moreover, in animal models, treatment with PARPi is not associated with genetic instability or cancer development in normal cells. This difference in bio-genetic response between BRCA-mutant tumor cells and normal cells when exposed to PARPi, would indicate that these agents are expected to be less toxic and much more specific than standard cytotoxic chemotherapy (26).

The majority of PARPi known to date have been developed as NAD competitors and they all function as potent blockers of the PARP1 and 2 enzymes. As these agents inhibit the catalytic action of PARP enzymes, they subsequently prevent the autoPARylation reactions, thereby preventing repair of DNA SSB. Moreover, PARPi also prevent PARP enzyme dissociation from the DNA (also known as PARP trapping on DNA lesions), which is highly toxic a process in HR-deficient cells $(17,27)$. The 5 clinically developed PARP inhibitors (olaparib, niraparib, rucaparib, talazoparib, and veliparib), have an extremely variable potential to exert PARP trapping and PARP catalytic inhibition $(28,29)$. For instance, the difference between Talazoparib and Olaparib (the 2 approved PAPRi in mBC) is up to 100 -fold in the trapping potency, and only 3 -fold in the catalytic potency (in favor of Talazoparib in either case). Although PARP trapping has been shown to strongly contribute to cytotoxicity of PARPi on cancer cells, this therapeutic advantage is markedly offset by its parallel toxic effects on the healthy bone marrow cells, which necessitated the clinical use of much lower doses of PARPi with high trapping potency. This would explain why Olaparib is used at $300 \mathrm{mg}$ (twice per day), while talazoparib is used at I mg (once per day) (30,31). However, and irrespective to the drug potency and therapeutic doses, the five clinically developed PAPRi, had shown some class effect adverse events, related mainly to NAD+ inhibition. Nausea and fatigue are the most common toxicities being encountered in around $40-50 \%$ of the PARPi treated patients regardless of the agent used. However, the frequency of hematological 
toxicity is quite variable, being highest with talazoparib and niraparib, and lowest with veliparib (32-35). MDS/AML has been also reported in $<1 \%$ in patients treated by niraparib (0.9\%), Olaparib (0.8\%), and rucaparib (0.5\%) (36-38). Of note, all of these patients had been previously treated with platinum-based received chemotherapy, which can also induce DNA repair defects, therefore it is not completely clear whether PARPi directly caused MDS/AML or it is the joint effects of PARP inhibition and cytotoxic agents (35). Finally, as PARPi are functioning via inhibition of DNArepair, hence their clinical use may be also associated with increased rate of intra-tumoral genomic mutations. These continuously evolving mutations may induce partial restoration of HRR pathway (the original target of PARPi), leading to evolution of resistant clones and subsequent treatment failure with their long-term use.

\section{Clinical studies of PAPRi in metastatic breast cancer (MBC)}

Initially, the clinical interest in PARPi was largely directed to patients with high-grade serous ovarian cancer (HGSOC), in view of the high incidence of HRR defects (HRD) among these patients, related to the presence BRCA1/2 germline mutations (found in around $15 \%$ ), or the presence other genetic , somatic or epigenetic defects in HRR pathway (found in additional $30-35 \%$ ). HRD is an important therapeutic target in HGSOC as shown by the high efficacy of platinums and PARPi in this disease (39). At the present time 3 PARPi (olaparib, niraparib, rucaparib) are approved as a maintenance therapy in platinum sensitive relapse (40-42), while Olaparib has been also approved as maintenance therapy following response to first-line platinum-based chemotherapy (43).

On the contrary, the incidence of germline, somatic and epigenetic HRD in breast cancer women is much less than those with ovarian cancer (around 10\% in all breast cancer patients, with almost half of them harboring germline BRCA mutations). Given the relatively high prevalence of HRD in TNBC (44), the use of PARPi has initially emerged as an exciting treatment option for these patients irrespective to their BRCA mutation status. The first randomized phase II study of the combination of gemcitabine and carboplatin with or without iniparib, showed improvement in overall survival over chemotherapy alone, yet these results were not confirmed in a subsequent phase III trial (45). The current evidence clearly indicates that these agents have weak activity in unselected TNBC and hence the importance of focusing on patients with germline BRCA mutations as a definite exemplar for the presence of HRD. Two early proof of concept trials, had reported an ORR of $41 \%$ and $50 \%$ with Olaparib and talazoparib respectively in $\mathrm{mBC}$ patients with germline BRCA mutations $(46,47)$. Importantly, both agents were equally effective in TNBC or $\mathrm{HR}+$ disease, which confirmed the hypothesis that PARPi are typically genotype-specific rather than phenotype-specific therapy.

In view of the above, 2 phase III randomized studies of similar design (OlympiAD and EMBRACA) were successively conducted to test the efficacy and safety of these 2 agents versus single agent chemotherapy [physician's choice of therapy (PCT) among several non-platinum agents) in HER2 negative $\mathrm{mBC}$ patients with germline BRCA mutations. In the OlympiAD trial $(\mathrm{N}=302)$, patients treated with olaparib showed a median PFS of 7.0 vs. 4.2 months for those treated with single-agent chemotherapy (HR: $0.58 ; \mathrm{P}<0.001)(31)$. The ORR was also markedly higher with olaparib compared to chemotherapy $(59.9 \%$ vs. $28.8 \%$, respectively). Likewise, in the EMBRACA trial $(\mathrm{N}=431)$, talazoparib could provide a significantly longer median PFS than chemotherapy (8.6 vs. 5.6 months, respectively; HR: $0.54 ; \mathrm{P}<0.001)$ and a much higher ORR $(62.6 \%$ vs. $27.2 \%$, for talazoparib and chemotherapy respectively) (30). Strikingly, both PARPi were able to induce tumor response at a short time, comparable to that achieved by chemotherapy (Table 1).

At median follow-up period of 2 years, there was no statistically significant improvement in OS with olaparib compared to TPC at, however, in a prespecified subgroup analysis, there was a meaningful OS benefit in the olaparib arm compared to PCT arm in patients with no prior chemotherapy for metastatic disease (median OS of 22.6 and 14.7 months respectively, HR 0.51 (95\% CI: 0.29-0.90; $\mathrm{P}=0.02$ ) (48). For the EMBRACA study, the median OS in patients treated by Talazoparib and PCT were 19.3 and 19.5 months respectively (HR $=0.85 ; 95 \%$ CI: $0.67-1.07$; $\mathrm{P}=0.17)$. Although, at the first glance the efficacy data of these 2 PARPi versus chemotherapy may look quite similar, yet in the EMBRACA study there was a significant prolongation of response duration in talazoparib treated patients compared to PCT (5.4 vs. 3.1 months respectively $\mathrm{HR}=0.43,95 \%$ CI: $0.27-0.70$ ), which was not the case with olaparib in the OlympiAD study.

In general, treatment with olaparib and Talazoparib was relatively well tolerated, with fatigue, anemia, nausea, and vomiting (mostly grade I-II) being the most common adverse events encountered with the 2 agents. The rate of 
Table 1 Comparison between efficacy and safety outcomes of the OlympiAD and EMBRACA studies

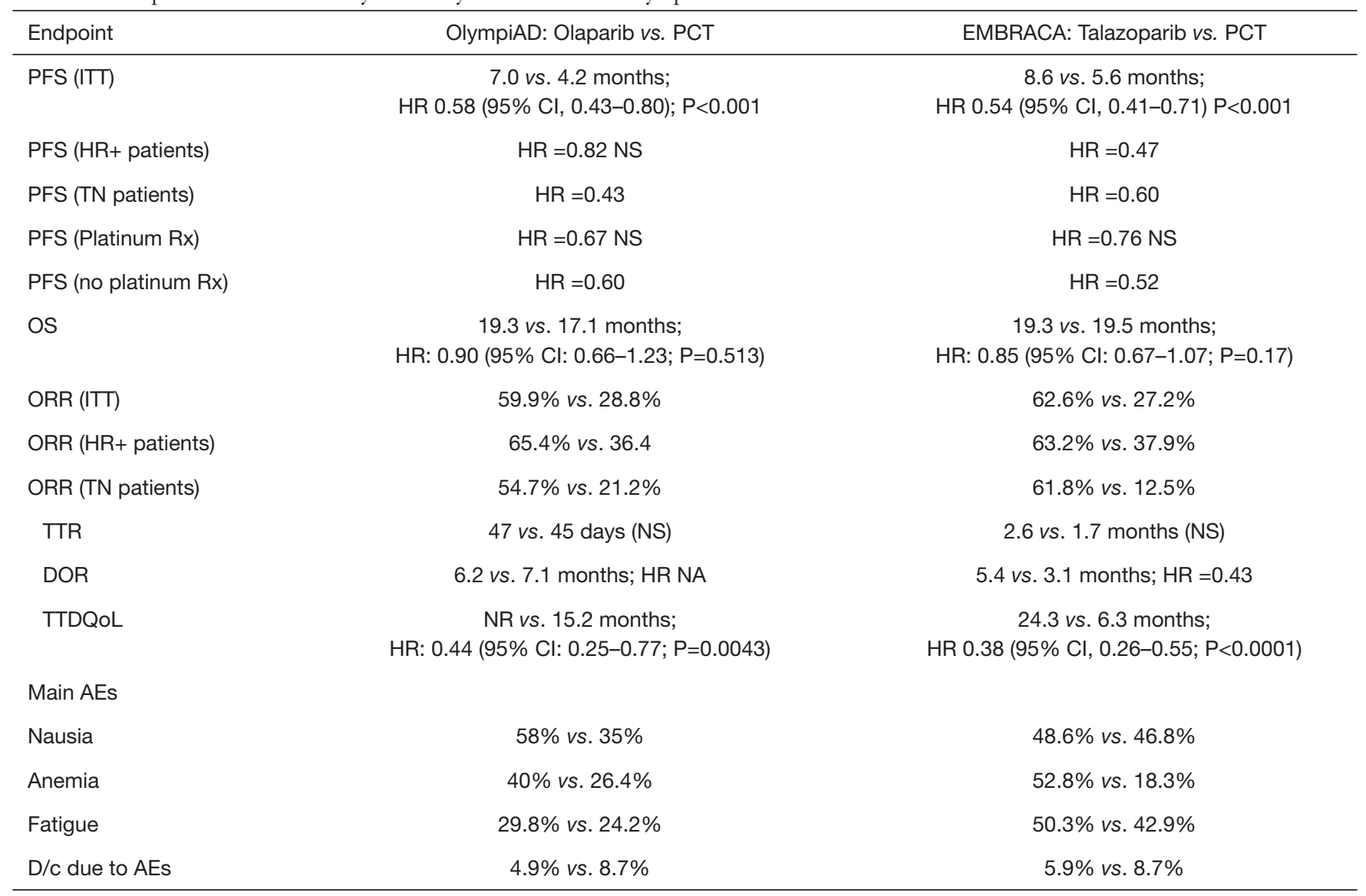

PFS, progression free survival; OS, overall survival; ORR, overall response rate; TN, triple negative; HR+, hormone receptor positive; TTDQoL, time to deterioration in global HRQoL; DOR, duration of response; TTR, time to response; AEs, adverse events; D/c, treatment discontinuation; NS, not significant; NR, not reached; HR, hazard ratio.

treatment discontinuation because of adverse events was lower than that reported in patients treated with single agent chemotherapy $(30,31)$. Of note, in the 2 studies, there was a highly significant prolongation in the time to deterioration of global HRQoL, among patients treated with the 2 PARP inhibitors compared to those treated with PCT, which is another extremely relevant advantage of these agents compared to single agent chemotherapy (Table 1).

\section{Platinums versus PARPi in MBC}

As mentioned earlier, and in alignment with PARPi mechanism of action, HRR is also needed to repair DNA lesions caused by platinum salts, and hence the expected preferential benefit of these agents among patients with BRCA mutations. It is worth mentioning that platinums and PARPi do not share the exact mechanisms of action and resistance. For instance, the platinum-induced DNA lesions occurs in the form of complex cross-links, which are more lethal than the SSBs caused by PARPi. Although the use of either class is associated with genetic alterations in the tumor cells, that may lead to restoration of HRR in BRCA1-mutated tumor, nevertheless, tumor cells with restoration of HRR may still retain sensitivity to platinum , while exhibiting resistance to PARP inhibitors (32).

The interest of platinum salts have recently emerged in patients with mTNBC, in spite of the fact that carboplatin was not found to be superior to docetaxel in the overall population of mTNBC patients, as reported in the TNT study. Importantly, in this randomized phase III study, carboplatin could double the ORR compared to docetaxel onlyin patients with gBRCA [68\% vs. $33 \%$ respectively, test for interaction $(\mathrm{P}=0.01)](25)$. While, these results were generated on a small subset of 43 patients and thus 
should be interpreted with caution, yet they appear largely comparable to what was reported with the PARPi in the OlympiAD and EMBRACA studies. This would strongly suggest a genotype-specific nature of platinum cytotoxicity in BRCA-mutant breast cancer.

Notably, earlier results from ovarian cancer studies have clearly demonstrated that resistance to PARPi is so much related to resistance to prior platinum therapy. In a small study, which included 50 patients treated by olaparib for their relapsing OC, Fong et al. have reported an ORR of $15 \%$ among patients with platinum-refractory disease (defined as progression while receiving platinum or within 0-3 months after the last dose of platinum), and $40 \%$ for those with platinum-resistant disease (patients who progressed within 3-6 months after the last dose of platinum) (49). In the same report, patients with platinum sensitive disease (patients who progressed after $>6$ months) had the maximum benefit of olaparib treatment with an ORR of $60 \%$. In line with OC data, the ABRAZO 2-cohort study could further confirm that platinum sensitivity (progression after $>6$ months) is a strong predictor to ensuing sensitivity to PARPi treatment in $\mathrm{mBC}$. This phase II study included one cohort of $49 \mathrm{mBC}$ patients who received previous platinum treatment, with a median time from the last platinum dose to disease progression of 4 months (range, 0.03-49.15 months) (50). All patients received $1 \mathrm{mg}$ of talazoparib once daily as continuous therapy until disease progression or unacceptable toxicity. In the whole cohort, the ORR was quite modest $(21 \%)$, however in the subgroup of patients with platinum-free interval $>6$ months, the ORR reached up to $48 \%$, versus $10 \%$ in the remaining patients who progressed within 0-6 months following the last dose of platinum (50). The OlympiAD and EMBRACA studies have included a minority of patients who were previously treated with a platinum agent $(21 \%$ and $16 \%$ in OlympiAD and EMBRACA respectively). In both studies, a clinical evidence of platinum sensitivity was required for patients' inclusion (30,31). Nevertheless, the efficacy data of PARPi in platinum pre-treated patients was less pronounced in both studies, although it was still nonsignificantly superior to PCT (Table 1). Taken together, it is clear that sensitivity to prior platinum therapy (defined as no disease progression for $>6$ months) appears as an important clinical biomarker to predict sensitivity to PARPi in platinum-pretreated patients, which strongly suggests the presence of a cross-resistance between platinums and PARPi.

\section{Integrating PARPi into the treatment algorithm of BRCA-mutated MBC patients}

In BRCA mutant mTNBC first-line PARP inhibitors should certainly be considered in the majority of patients, who completed adjuvant anthracycline-taxane therapy for their early-stage disease. As mentioned earlier, single agent carboplatin appears to provide a closely similar therapeutic benefit in these patients, however it is unclear which agent should be used first. While there is no head-to-head data comparing platinums versus PARPi in patients with MBC and germline BRCA1/2 mutations, yet we believe that the data concerning the safety profile and quality of life is quite convincing in favor of initial PARPi (any of the 2 approved agents). In such case if the patients responds to this treatment, we may consider subsequent platinum therapy at the time of disease progression. Although this sequence is not really evidence based, still it is an extrapolation from some OC studies reporting responses to platinums in Olaparibresistant, heavily pretreated BRCA1/2-mutated patients. On the other hand, patients with HR+/HER2- BRCA-mutated $\mathrm{MBC}$ should be treated with endocrine therapy preferably in combination with a CDK4/6 inhibitor, which would certainly provide a much more effective therapy, with a more favorable toxicity profile compared to PARPi. In the event of disease progression after $1^{\text {st }}$ line ET with a CDK4/6 inhibitor other ET options in the second-line setting may be also considered before a PARPi is to be offered. An exceptional situation may be in a patients progressing after a CDK4/6 inhibitor, with severe disease-related symptoms, where PARPi with their high response rate and rapid time to response will be needed to achieve a prompt palliation.

\section{Future directions in PARP inhibitors in breast cancer}

In light of the rather overlapping mechanism of actions, key questions need to be addressed: First, Would the addition of PARPi to platinum improve patients' outcome? And Second, would a head to head comparison show superiority of one agent over the other? Third, what would be the perfect future combination partner with PARP inhibitors specially in TNBCs?

\section{PARPi in combination with platinum based chemotherapy in BRCA mutant breast cancer}

Both platinums and PARPi have mutual mechanisms of resistance, which suggest their use in combination 
Table 2 Studies using Combination of veliparib + carboplatin/paclitaxel chemotherapy versus placebo + carboplatin/paclitaxel chemotherapy in early and advanced breast cancer women

\begin{tabular}{|c|c|c|c|c|c|c|c|c|c|c|}
\hline Study & $\mathrm{N}$ & Phase & $\begin{array}{l}\text { Disease } \\
\text { setting }\end{array}$ & $\begin{array}{c}\% \\
\text { gBRCA } \\
\text { positive }\end{array}$ & PARPi dose & Chemo regimen & $\begin{array}{l}\% \text { pCR } \\
\text { vs. Pla }\end{array}$ & $\begin{array}{l}\% \text { ORR } \\
\text { vs. Pla }\end{array}$ & $\begin{array}{c}\text { mPFS in } \\
\text { mos vs. Pla }\end{array}$ & $\begin{array}{c}\text { mOS in } \\
\text { mos vs. Pla }\end{array}$ \\
\hline BROCKADE 3 & 512 & $\begin{array}{c}\text { Phase III; } \\
\text { randomized } \\
(2: 1)\end{array}$ & $\mathrm{aBC}$ & $100 \%$ & $\begin{array}{l}\text { V } 120 \text { mg PO } \\
\text { bid; D-2 to } \\
\text { D5/3 weeks }\end{array}$ & $\begin{array}{c}\text { C: AUC6/3 wks. } \\
\text { P: } 80 \mathrm{mg} / \mathrm{m}^{2} / \text { week }\end{array}$ & NA & $\begin{array}{l}75.8 \% \text { vs. } \\
74 \% \text {; NS }\end{array}$ & $\begin{array}{c}14.5 \text { vs. } \\
12.6 ; \\
P=0.002\end{array}$ & $\begin{array}{l}33.5 \text { vs. } \\
28.2 ; \text { NS }\end{array}$ \\
\hline
\end{tabular}

*, 316 patients randomized to paclitaxel plus carboplatin plus veliparib, 160 patients randomized to paclitaxel plus carboplatin plus placebo, (and 158 patients randomized to paclitaxel alone, their data are not shown in Table 2); **, 97 patients randomized to paclitaxel plus carboplatin plus veliparib, 99 patients randomized to paclitaxel plus carboplatin plus placebo (and 94 patients randomized to temozolomide and veliparib, their data are not shown in Table 2). P, paclitaxel; C, carboplatin; V, veliparib; Pla, placebo; pCR, pathological complete remission; ORR, overall response rate; PFS, progression free survival; OS, overall survival; Mos, months; Wk, week; NS, not significant; aBC, advanced breast cancer; Neoadj, neoadjuvant.

rather than sequentially. In preclinical models of BRCA1deficient mammary tumors, the combination of platinums and PARPi inhibitor was shown to be strongly synergistic, which supported their combined use in the clinic (51). Nonetheless, the presence of many overlapping toxicities (including nausea, anemia and others), have prohibited the use of the standard continuous dosing of PARPi in combination with platinums or other cytotoxic agents. Among all PARPi, veliparib has shown the least incidence of bone marrow toxicity and hence it emerged as a potentially suitable agent to safely combine with chemotherapy. Three randomized studies have addressed the role of adding veliparib to carboplatin/paclitaxel doublet (CP) in women with breast cancer (Table 2).

The BrighTNess phase III study tested the value of neoadjuvant veliparib (at $50 \mathrm{mg}$ BID orally on days $1-7 / 3$ weeks) versus placebo, when combined with $3-4$ cycles of neoadjuvant $\mathrm{CP}$ in women with operable TNBC. All patients subsequently received 4 cycles of the AC regimen which was followed by loco-regional surgery. The pCR rate was the primary end point of the study. In the overall study population, there was no improvement in the pCR rate with veliparib compared to placebo (53\% and $58 \%$ respectively) (52). While the results of the BrighTNess study are bluntly negative, it is important to note that this study was conducted in unselected TNBC patients and not exclusively in patients with BRCA mutations, who are the unique responders to PARPi. However, in the subgroup of patients with BRCA mutations (15\% of the total study population), there was a numerical increase of the $\mathrm{pCR}$ rate in veliparib arm compared to placebo arm (57\% and 50\% respectively). While subgroups analysis was negative for interaction according to BRCA status, this could be down to low statistical power. In addition, it is also arguable that the dose of veliparib used in this study (50 mg BID orally on days $1-7 / 3$ weeks), is far below its standard dose when given as a single agent (300-400 mg BID orally, continuous dosing). Obviously such extremely low dose of veliparib may not provide a meaningful therapeutic PARP inhibition in the treated patients (52).

The other 2 studies, the BROCADE- 2 and the BROCADE-3 $(53,54)$, have investigated the role of a higher dose of veliparib (120 mg BID orally for 7 days/3 weeks), when combined with the $\mathrm{CP}$ regimen, in patients with advanced breast cancer who received $\leq 2$ prior lines of cytotoxic therapy for MBC. Unlike the BrighTNess study, all patients included in the 2 BROCADE studies had germline BRCA mutations. In the 2 studies the primary end point was PFS and the secondary end points included OS and ORR. In the BROCADE-2 phase II study, the median PFS was 14.1 and 12.3 months, in the veliparib and placebo arms respectively [HR 0.789 ; 95\% CI, $0.536-$ 1.162; $\mathrm{P}=0.227]$, and the interim median $\mathrm{OS}$ was 28.3 and 25.9 months (HR 0.750; 95\% CI, 0.503-1.117; $\mathrm{P}=0.156$ ). 
Notably the ORR was significantly higher in the veliparib arm compared to placebo ( $77.8 \%$ and $61.3 \%$ respectively, $\mathrm{P}=0.027$ ) (53). Although BROCADE-2 has also failed to achieve its primary end point, it could actually provide a positive signal for some efficacy benefits of veliparib in such dose schedule when combined with CP.

The much larger BROCADE-3 phase III study subsequently demonstrated a significant improvement in median PFS with veliparib versus placebo (14.5 vs. 12.6 months respectively $(\mathrm{HR}=0.71, \mathrm{P}=0.002)$. Although the ORR was similarly high in both arms $(75.8 \%$ and $74.1 \%$ respectively), however duration of response was longer in the veliparib arm compared to placebo arm (14.7 vs. 11 months respectively $\mathrm{P}$ value not available). Importantly, at 3 years, $26 \%$ of patients randomized to veliparib were alive and progression free compared to only $11 \%$ of patients in the control arm (54). As expected, the addition of veliparib was associated with more hematological toxicity, yet this did not appear to considerably impact compliance to therapy and overall patient tolerance. The results of the BROCADE-3 may represent a paradigm shift in managing patients with BRCA-mutant advanced breast cancer, in view of the impressive $15 \%$ absolute difference in the 3 yearPFS, which was achieved by the veliparib, versus placebo when combined with $\mathrm{CP}$ (54).

\section{PARPi in combination with paclitaxel in early stage breast cancer}

As mentioned earlier both the OlympiAD and EMBRACA randomized patients to either a PARPi or investigator choice of several chemotherapeutics but not platinum. Direct comparison between PARPi and platinums was possibly "consciously" avoided in the 2 PARPi pivotal studies during the metastatic setting. The $1^{\text {st }}$ head to head study comparing PARPi versus platinums was conducted in patients treated during the neoadjuvant setting (GeparOLA trial). This phase II trial included patients who had HER2-negative breast cancer and HRR deficiency (defined as having a high HRD score or a germline or a somatic BRCA1/2 mutation; $\mathrm{n}=102$ ). These patients were randomized to receive either 12 weeks of paclitaxel ( $80 \mathrm{mg} / \mathrm{m}^{2} /$ week) plus olaparib $100 \mathrm{mg}$ BID twice daily, or the same dose of paclitaxel plus carboplatin (AUC 2/week). All patients were then treated by 4 cycles of epirubicin/ cyclophosphamide, which was followed by surgery (55). Although there was no significant difference in the pCR rate between olaparib and carboplatin arms (55.1\% vs. $48.6 \%$ respectively), however in a predefined subgroup analyses, patients who were hormone receptor positive $(n=32)$ had a remarkable difference in the pCR rate favoring olaparib versus carboplatin ( $52 \%$ vs. or $20 \%$ respectively). A similar advantage of olaparib was also observed among women younger than 40 years of age $(n=29)$, in whom the $\mathrm{pCR}$ rate was $76 \%$ and $45 \%$ in the 2 arms respectively. Of a special interest, in the subgroup of patients without germline or somatic BRCA mutations (45 patients), the olaparib arm has also shown a superior pCR rate compared to carboplatin (51\% vs. $37 \%$ respectively) (55). Should this particular finding be validated in future trials, then the number of breast cancer patients benefiting from olaparib will further expand to include all patients whose tumors harbor other HRD beyond BRCA mutations. In all other subgroups, the pCR rates were quite similar across the 2 treatment arms. Importantly, a much less rate of serious adverse events was reported in olaparib arm compared to carboplatin arm $(13 \%$ and 51\% respectively). While the GeparOLA trial should be saluted to show for the 1st time that olaparib/paclitaxel combination is a safe and effective regimen in breast cancer patients harboring HRD, still the reasons behind the observed superior outcome with olaparib versus carboplatin in certain subgroups are difficult to explain. Hence these results should be only considered as hypothesis generating, that need further exploration in a larger trial.

\section{PARP inhibitors in combination with immunotherapy}

Another venue is to combine PARPi with ani-PD1/PDL1. The latter is currently approved in managing TNBC when given with front line chemotherapy. Preclinical rational pointed out to the potential synergy when combining them with PARPi. It was shown that the genomic instability and DNA damage induced by PARPi is able to increase tumor immunogenicity via expression of more neoantigens by the tumor cells (56). Such DNA damage was also found to up-regulate PDL1 by inducing interferon expression and activation of innate immune pathways $(57,58)$. Recently, data from a phase I/b study testing the combination of the PARPi, pamiparib, and a humanized anti-PD1, showed a promising response rate of $20 \%$, with stable disease in $32 \%$, in heavily pretreated patients with advanced solid tumors irrespective of their BRCA status (59). This study included 49 patients, and it remains premature to judge the clinical efficacy and safety of the combination, but several other studies are currently looking into that. Obviously, it would be relevant to adjust the dose and schedule of this 
potentially promising combination to achieve maximum benefit without compromising patient safety. Finally, a recent phase II single arm study combined olaparib and the PD-L1 inhibitor durvalumab in advanced breast cancer patients with gBRCA mutation (60). Out of the recruited 30 patients, $63.3 \%$ achieved objective response which reached $70 \%$ in patients who received $0-1$ prior line of therapy. Median PFS was 8.2 months in the whole cohort and 11.7 months in those with $0-1$ prior lines. Such promising chemo-free combination needs further exploration in randomized studies.

\section{PARP inbibitors in early stage breast cancer}

Currently, the OLYMPIA trial is testing the role of adjuvant olaparib in patients with BRCA mutations. This trial has already completed the enrollment of around 1,800 patients with primary results expected by end of 2020 . In the neoadjuvant setting, single agent talazoparib setting showed a promising $53 \%$ PCR rate $(61)$. The residual cancer burden class $0 / \mathrm{I}(\mathrm{RCB} 0 / \mathrm{I})$ was $63 \%$ with relatively accepted safety profile.

\section{Conclusions and future directions}

PARPi have established themselves as a cornerstone treatment in managing patients with advanced BRCA mutated breast cancer. Although the combination of PARPi with platinums and other agents seems promising, we still need additional data to reach firm conclusions for their therapeutic utility.

\section{Acknowledgments}

Funding: None.

\section{Footnote}

Provenance and Peer Review: This article was commissioned by the Guest Editor (Shaheenah Dawood) for the series "Targeting the DNA Damaging Pathway: PARPi and Beyond" published in Chinese Clinical Oncology. The article has undergone external peer review.

Conflicts of Interest: All authors have completed the ICMJE uniform disclosure form (available at http://dx.doi. org/10.21037/cco-19-230). The series "Targeting the DNA Damaging Pathway: PARPi and Beyond" was commissioned by the editorial office without any funding or sponsorship. Dr. LK reports personal fees from Pfizer Oncology, personal fees from Roche Oncology, personal fees from Novartis, outside the submitted work. Dr. HA Jr reports personal fees from Roche, personal fees from Novartis, personal fees from Innate Pharma, outside the submitted work. The authors have no other conflicts of interest to declare.

Ethical Statement: The authors are accountable for all aspects of the work in ensuring that questions related to the accuracy or integrity of any part of the work are appropriately investigated and resolved.

Open Access Statement: This is an Open Access article distributed in accordance with the Creative Commons Attribution-NonCommercial-NoDerivs 4.0 International License (CC BY-NC-ND 4.0), which permits the noncommercial replication and distribution of the article with the strict proviso that no changes or edits are made and the original work is properly cited (including links to both the formal publication through the relevant DOI and the license). See: https://creativecommons.org/licenses/by-nc-nd/4.0/.

\section{References}

1. Walsh T, Lee MK, Casadei S, et al. Detection of inherited mutations for breast and ovarian cancer using genomic capture and massively parallel sequencing. Proc Natl Acad Sci U S A 2010;107:12629-33.

2. Stratton MR, Rahman N. The emerging landscape of breast cancer susceptibility. Nat Genet 2008;40:17-22.

3. Economopoulou P, Dimitriadis G, Psyrri A. Beyond BRCA: new hereditary breast cancer susceptibility genes. Cancer Treat Rev 2015;41:1-8.

4. Anders C, Carey LA. Understanding and treating triplenegative breast cancer. Oncology (Williston Park) 2008;22:1233-9; discussion 9-40, 43.

5. Lehmann BD, Bauer JA, Chen X, et al. Identification of human triple-negative breast cancer subtypes and preclinical models for selection of targeted therapies. J Clin Invest 2011;121:2750-67.

6. Mavaddat N, Barrowdale D, Andrulis IL, et al. Pathology of breast and ovarian cancers among BRCA1 and BRCA2 mutation carriers: results from the Consortium of Investigators of Modifiers of BRCA1/2 (CIMBA). Cancer Epidemiol Biomarkers Prev 2012;21:134-47.

7. Schneider BP, Winer EP, Foulkes WD, et al. Triple- 
negative breast cancer: risk factors to potential targets. Clin Cancer Res 2008;14:8010-8.

8. Hoeijmakers JH. Genome maintenance mechanisms for preventing cancer. Nature 2001;411:366-74.

9. Helleday T. Homologous recombination in cancer development, treatment and development of drug resistance. Carcinogenesis 2010;31:955-60.

10. Konstantinopoulos PA, Ceccaldi R, Shapiro GI, et al. Homologous Recombination Deficiency: Exploiting the Fundamental Vulnerability of Ovarian Cancer. Cancer Discov 2015;5:1137-54.

11. Helleday T, Petermann E, Lundin C, et al. DNA repair pathways as targets for cancer therapy. Nat Rev Cancer 2008;8:193-204.

12. Aguilar-Quesada R, Munoz-Gamez JA, Martin-Oliva D, et al. Interaction between ATM and PARP-1 in response to DNA damage and sensitization of ATM deficient cells through PARP inhibition. BMC Mol Biol 2007;8:29.

13. Buisson R, Dion-Cote AM, Coulombe Y, et al. Cooperation of breast cancer proteins PALB2 and piccolo BRCA2 in stimulating homologous recombination. Nat Struct Mol Biol 2010;17:1247-54.

14. Rafnar T, Gudbjartsson DF, Sulem P, et al. Mutations in BRIP1 confer high risk of ovarian cancer. Nat Genet 2011;43:1104-7.

15. Farmer H, McCabe N, Lord CJ, et al. Targeting the DNA repair defect in BRCA mutant cells as a therapeutic strategy. Nature 2005;434:917-21.

16. Chaudhuri AR, Nussenzweig A. The multifaceted roles of PARP1 in DNA repair and chromatin remodelling. Nat Rev Mol Cell Biol 2017;18:610-21.

17. Murai J, Huang SY, Das BB, et al. Trapping of PARP1 and PARP2 by Clinical PARP Inhibitors. Cancer Res 2012;72:5588-99.

18. Mateo J, Lord CJ, Serra V, et al. A decade of clinical development of PARP inhibitors in perspective. Ann Oncol 2019;30:1437-47.

19. Valerie K, Povirk LF. Regulation and mechanisms of mammalian double-strand break repair. Oncogene 2003;22:5792.

20. Wang ZQ, Auer B, Stingl L, et al. Mice lacking ADPRT and poly(ADP-ribosyl)ation develop normally but are susceptible to skin disease. Genes Dev 1995;9:509-20.

21. Helleday T. The underlying mechanism for the PARP and BRCA synthetic lethality: clearing up the misunderstandings. Mol Oncol 2011;5:387-93.

22. Harvey A, Mielke N, Grimstead JW, et al. PARP1 is required for preserving telomeric integrity but is dispensable for A-NHEJ. Oncotarget 2018;9:34821-37.

23. Bryant HE, Schultz N, Thomas HD, et al. Specific killing of BRCA2-deficient tumours with inhibitors of poly(ADPribose) polymerase. Nature 2005;434:913-7.

24. Isakoff SJ. Triple-negative breast cancer: role of specific chemotherapy agents. Cancer J 2010;16:53-61.

25. Tutt A, Tovey H, Cheang MCU, et al. Carboplatin in BRCA1/2-mutated and triple-negative breast cancer BRCAness subgroups: the TNT Trial. Nat Med 2018;24:628-37.

26. Annunziata CM, O'Shaughnessy J. Poly (ADP-ribose) polymerase as a novel therapeutic target in cancer. Clin Cancer Res 2010;16:4517-26.

27. Hopkins TA, Shi Y, Rodriguez LE, et al. Mechanistic Dissection of PARP1 Trapping and the Impact on In Vivo Tolerability and Efficacy of PARP Inhibitors. Mol Cancer Res 2015;13:1465-77.

28. Murai J, Huang SY, Renaud A, et al. Stereospecific PARP trapping by BMN 673 and comparison with olaparib and rucaparib. Mol Cancer Ther 2014;13:433-43.

29. Shen Y, Aoyagi-Scharber M, Wang B. Trapping Poly(ADP-Ribose) Polymerase. J Pharmacol Exp Ther 2015;353:446-57.

30. Litton JK, Rugo HS, Ettl J, et al. Talazoparib in Patients with Advanced Breast Cancer and a Germline BRCA Mutation. N Engl J Med 2018;379:753-63.

31. Robson M, Im SA, Senkus E, et al. Olaparib for Metastatic Breast Cancer in Patients with a Germline BRCA Mutation. N Engl J Med 2017;377:523-33.

32. Bitler BG, Watson ZL, Wheeler LJ, et al. PARP inhibitors: Clinical utility and possibilities of overcoming resistance. Gynecol Oncol 2017;147:695-704.

33. Staropoli N, Ciliberto D, Del Giudice T, et al. The Era of PARP inhibitors in ovarian cancer: "Class Action" or not? A systematic review and meta-analysis. Crit Rev Oncol Hematol 2018;131:83-9.

34. Bao Z, Cao C, Geng X, et al. Effectiveness and safety of poly (ADP-ribose) polymerase inhibitors in cancer therapy: A systematic review and meta-analysis. Oncotarget 2016;7:7629-39.

35. LaFargue CJ, Dal Molin GZ, Sood AK, et al. Exploring and comparing adverse events between PARP inhibitors. Lancet Oncol 2019;20:e15-e28.

36. Ledermann J, Harter P, Gourley C, et al. Olaparib maintenance therapy in platinum-sensitive relapsed ovarian cancer. N Engl J Med 2012;366:1382-92.

37. Mirza MR, Monk BJ, Herrstedt J, et al. Niraparib Maintenance Therapy in Platinum-Sensitive, Recurrent 
Ovarian Cancer. N Engl J Med 2016;375:2154-64.

38. Coleman RL, Oza AM, Lorusso D, et al. Rucaparib maintenance treatment for recurrent ovarian carcinoma after response to platinum therapy (ARIEL3): a randomised, double-blind, placebo-controlled, phase 3 trial. Lancet 2017;390:1949-61.

39. Konstantinopoulos PA, Ceccaldi R, Shapiro GI, et al. Homologous recombination deficiency: exploiting the fundamental vulnerability of ovarian cancer. Cancer Discov 2015;5:1137-54.

40. Ison G, Howie LJ, Amiri-Kordestani L, et al. FDA approval summary: Niraparib for the maintenance treatment of patients with recurrent ovarian cancer in response to platinum-based chemotherapy. Clin Cancer Res 2018;24:4066-71.

41. Balasubramaniam S, Beaver JA, Horton S, et al. FDA Approval Summary: Rucaparib for the Treatment of Patients with Deleterious BRCA MutationAssociated Advanced Ovarian Cancer. Clin Cancer Res 2017;23:7165-70.

42. Kim G, Ison $\mathrm{G}, \mathrm{McKee} \mathrm{AE}$, et al. FDA approval summary: olaparib monotherapy in patients with deleterious germline BRCA-mutated advanced ovarian cancer treated with three or more lines of chemotherapy. Clin Cancer Res 2015;21:4257-61.

43. Moore K, Colombo N, Scambia G, et al. Maintenance Olaparib in Patients with Newly Diagnosed Advanced Ovarian Cancer. N Engl J Med 2018;379:2495-505.

44. Timms KM, Abkevich V, Hughes E, et al. Association of BRCA1/2 defects with genomic scores predictive of DNA damage repair deficiency among breast cancer subtypes. Breast Cancer Res 2014;16:475.

45. O'Shaughnessy J, Schwartzberg L, Danso MA, et al. Phase III study of iniparib plus gemcitabine and carboplatin versus gemcitabine and carboplatin in patients with metastatic triple-negative breast cancer. J Clin Oncol 2014;32:3840-7.

46. Tutt A, Robson M, Garber JE, et al. Oral poly (ADPribose) polymerase inhibitor olaparib in patients with BRCA1 or BRCA 2 mutations and advanced breast cancer: a proof-of-concept trial. Lancet 2010;376:235-44.

47. Turner NC, Telli ML, Rugo HS, et al. Final results of a phase 2 study of talazoparib (TALA) following platinum or multiple cytotoxic regimens in advanced breast cancer patients (pts) with germline BRCA1/2 mutations (ABRAZO). J Clin Oncol 2017;35:1007.

48. Robson ME, Tung N, Conte P, et al. OlympiAD final overall survival and tolerability results: Olaparib versus chemotherapy treatment of physician's choice in patients with a germline BRCA mutation and HER2-negative metastatic breast cancer. Ann Oncol 2019;30:558-66.

49. Fong PC, Yap TA, Boss DS, et al. Poly(ADP)-ribose polymerase inhibition: frequent durable responses in BRCA carrier ovarian cancer correlating with platinumfree interval. J Clin Oncol 2010;28:2512-9.

50. Turner NC, Telli ML, Rugo HS, et al. A Phase II Study of Talazoparib after Platinum or Cytotoxic Nonplatinum Regimens in Patients with Advanced Breast Cancer and Germline BRCA1/2 Mutations (ABRAZO). Clin Cancer Res 2019;25:2717-24.

51. Rottenberg S, Jaspers JE, Kersbergen A, et al. High sensitivity of BRCA1-deficient mammary tumors to the PARP inhibitor AZD2281 alone and in combination with platinum drugs. Proc Natl Acad Sci U S A 2008;105:17079-84.

52. Loibl S, O'Shaughnessy J, Untch M, et al. Addition of the PARP inhibitor veliparib plus carboplatin or carboplatin alone to standard neoadjuvant chemotherapy in triplenegative breast cancer (BrighTNess): a randomised, phase 3 trial. Lancet Oncol 2018;19:497-509.

53. Han HS, Dieras V, Robson M, et al. Veliparib with temozolomide or carboplatin/paclitaxel versus placebo with carboplatin/paclitaxel in patients with BRCA1/2 locally recurrent/metastatic breast cancer: randomized phase II study. Ann Oncol 2018;29:154-61.

54. Diéras VC, Han HS, Kaufman B, et al. LBA9Phase III study of veliparib with carboplatin and paclitaxel in HER2negative advanced/metastatic gBRCA-associated breast cancer. Ann Oncol 2019;30.

55. Fasching PA, Jackisch C, Rhiem K, et al. GeparOLA: A randomized phase II trial to assess the efficacy of paclitaxel and olaparib in comparison to paclitaxel/carboplatin followed by epirubicin/cyclophosphamide as neoadjuvant chemotherapy in patients (pts) with HER2-negative early breast cancer (BC) and homologous recombination deficiency (HRD). J Clin Oncol 2019;37:506.

56. Strickland KC, Howitt BE, Shukla SA, et al. Association and prognostic significance of BRCA1/2-mutation status with neoantigen load, number of tumor-infiltrating lymphocytes and expression of PD-1/PD-L1 in high grade serous ovarian cancer. Oncotarget 2016;7:13587-98.

57. Sen T, Rodriguez BL, Chen L, et al. Targeting DNA Damage Response Promotes Antitumor Immunity through STING-Mediated T-cell Activation in Small Cell Lung Cancer. Cancer Discov 2019;9:646-61.

58. Wang Z, Sun K, Xiao Y, et al. Niraparib activates 
interferon signaling and potentiates anti-PD-1 antibody efficacy in tumor models. Sci Rep 2019;9:1853.

59. Friedlander M, Meniawy T, Markman B, et al. Pamiparib in combination with tislelizumab in patients with advanced solid tumours: results from the dose-escalation stage of a multicentre, open-label, phase $1 \mathrm{a} / \mathrm{b}$ trial. Lancet Oncol 2019;20:1306-15.

60. Domchek S, Postel-Vinay S, Im S, et al. 11910 Phase II study of olaparib (O) and durvalumab (D)(MEDIOLA): updated results in patients (pts) with germline BRCAmutated (gBRCAm) metastatic breast cancer (MBC). Ann Oncol 2019. doi: 10.1093/annonc/mdz253.017.

61. Litton JK, Scoggins ME, Hess KR, et al. Neoadjuvant Talazoparib for Patients With Operable Breast Cancer With a Germline BRCA Pathogenic Variant. J Clin Oncol 2020;38:388-94.
Cite this article as: Azim HA, Kassem L, Azim H Jr. Integrating PARP inhibitors into the management of breast cancer: where are we? Chin Clin Oncol 2021;10(5):50. doi: $10.21037 /$ cco-19-230 